\title{
Temperature Study of CdZnTe Coplanar-Grid Detectors
}

\author{
Mark Amman, Member, IEEE, Julie S. Lee, and Paul N. Luke
}

\begin{abstract}
The coplanar-grid (CPG) and other electrononly detection techniques have made possible the use of CdZnTe-based detectors for gamma-ray spectroscopy when high efficiency, good energy resolution, and near room temperature operation are required. Despite the demonstrated potential of the technologies, widespread use remains hampered in part by the limited availability of the highly uniform CdZnTe material required for highresolution spectroscopy. However, it has been recently shown that mild cooling of CdZnTe CPG detectors can result in a significant improvement in the energy resolution of the detectors thereby allowing a wider range of material to be used for high-resolution applications. In this paper, we show that improved spectroscopic performance can consistently be achieved through a combination of detector cooling and increased detector bias. Energy resolutions of about $1 \%$ FWHM at $662 \mathrm{keV}$ for detector volumes up to $2.3 \mathrm{~cm}^{3}$ have been obtained at $20^{\circ} \mathrm{C}$. With the electronic noise subtracted, this amounts to an intrinsic resolution of $0.76 \%$. We also show that further cooling of the detectors to $-30^{\circ} \mathrm{C}$ leads to field polarization and a loss of spectroscopic performance.
\end{abstract}

\section{INTRODUCTION}

CdZnTe-based coplanar-grid (CPG) detectors [1]-[4] have

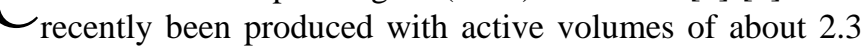
$\mathrm{cm}^{3}$ and energy resolutions near 1.6\% FWHM at $662 \mathrm{keV}$ [5], [6]. This represents a significant advancement in the technology. However, even such performance is still far from that expected based on simple theoretical modeling [7]. Several factors have been identified that currently limit the spectroscopic performance of CPG detectors [8]. These include electron trapping non-uniformity, electronic noise, and device non-uniformity. In order to further advance the technology, it is critical to better understand these degradation mechanisms and devise means to lessen or eliminate their impact.

We have recently shown that moderate cooling of CPG detectors can lead to improved spectroscopic performance [9].

Manuscript received February 28, 2006. This work was supported by the Director, Office of Science, of the U.S. Department of Energy under contract number DE-AC02-05CH-11231 and by the Director of the U.S. Department of Homeland Security.

The authors are with the Ernest Orlando Lawrence Berkeley National Laboratory, University of California, Berkeley, CA 94720 USA (telephone: 510-486-5638, e-mail: Mark_Amman@lbl.gov).
For this reason, we have performed a detailed study of the effects of reduced operating temperature on the performance characteristics of CPG detectors. The results of this study are summarized in this paper.

\section{EXPERIMENTAL METHOD}

The CdZnTe material used in this study was obtained from $\mathrm{eV}$ Products [10]. Two standard crystal sizes of approximately $10 \times 10 \times 10 \mathrm{~mm}^{3}$ and $15 \times 15 \times 10 \mathrm{~mm}^{3}$ were chosen because the sizes are representative of the current readily available CPG detector volumes. Prior to evaluating a crystal as a CPG detector, the material quality was verified using a standard set of characterization techniques [11]. These include the visual inspection of the crystal surfaces after lapping to detect any grain boundaries, transmission infrared microscopy through which pipes, precipitates, and inclusions are identified, and alpha particle response characterization of the crystal after fabrication into a simple planar detector. All of the crystals used in this study were free from random grain boundaries and significant quantities of large $(>20 \mu \mathrm{m}) \mathrm{Te}$ inclusions. Both of these defects are known to lead to charge transport non-uniformities that degrade the spectroscopic performance of the resultant gamma-ray detectors. The crystals used in this study also exhibited good overall uniformity in electron generation and transport as determined through the alpha particle measurements. Such uniformity is critical for achieving the best possible spectroscopic performance.

After the material characterization, the CdZnTe crystals were fabricated into CPG detectors using one of two processes. For both processes, the crystal surfaces were prepared by mechanically polishing with a water-based slurry of submicron alumina powder on a polishing pad followed by chemically etching in an approximately $2 \%$ bromine-methanol solution. Immediately following this etch, a full-area $\mathrm{Au}$ cathode was deposited onto one of the crystal surfaces through thermal evaporation. The subsequent formation of the grid electrodes differed between the two processes. In the conventional process, the grid electrodes were formed by thermally depositing Au through a shadow mask onto the surface opposing the cathode. Prior to the deposition, the surface was prepared by either a bromine-methanol etch or a chemical-mechanical polish. In the second process, to be referred to as the improved process, the grid electrodes were produced by first chemical-mechanically polishing the crystal surface followed by a full-area deposition of Au using thermal 
evaporation. This Au electrode was then photolithographically masked with the grid electrode pattern and patterned by sputter etching the exposed electrode areas. Any surface damage introduced by the sputtering was then removed by etching in bromine-ethylene glycol. As a final step, the detector was soaked in hydrogen peroxide in order to reduce the noise associated with the intergrid surface [12] and perhaps to improve the collection of charge to the collecting grid electrode [13].

Once a CPG detector was completed, it was loaded into a vacuum test chamber equipped with a thermoelectrically cooled detector holder. This allowed the detector temperature to be controlled from room temperature down to about $-40^{\circ} \mathrm{C}$. The detector electrodes were wired out to room temperature electronics so that a variety of measurements could be made. These measurements included intergrid leakage current, cathode leakage current, detector noise, and gamma-ray spectroscopic performance. The data was acquired as a function of temperature $\mathrm{T}$, cathode bias $-\mathrm{V}_{c}$, and collecting grid bias $\mathrm{V}_{\mathrm{g}}$. For the noise and gamma-ray measurements, the detector was operated using the differential-gain adjustment method to compensate for electron trapping [14]. A differential gain $G=1$ was used in the noise measurements. For the gamma-ray measurements, the values of $G, V_{c}$, and $V_{g}$ were optimized to obtain the best spectroscopic performance. Optimizing the performance of a detector by finely adjusting three independent parameters can be prohibitively time consuming particularly when this must be done for many detectors and for all experimental conditions. Consequently, we chose a more simplified optimization procedure consisting of testing the detector at both a typical $\mathrm{V}_{\mathrm{c}}$ of $1500 \mathrm{~V}$ and a much larger one of $3000 \mathrm{~V}$. At these two cathode biases, the detector was evaluated with $\mathrm{V}_{\mathrm{g}}$ ranging from $50 \mathrm{~V}$ to $200 \mathrm{~V}$ in steps of $50 \mathrm{~V}$. Then at each combination of $\mathrm{V}_{\mathrm{c}}$, and $\mathrm{V}_{\mathrm{g}}, \mathrm{G}$ was adjusted to obtain the best possible $662 \mathrm{keV}$ gamma-ray peak resolution.

\section{RESULTS}

Example spectra demonstrating the substantially improved performance that can be achieved through mild cooling of CdZnTe CPG detectors are shown in Figs. 1 and 2. Plotted in the figures are ${ }^{137} \mathrm{Cs}$ spectra obtained from two different detectors produced using our improved grid electrode fabrication process. Spectra obtained from a $10 \times 10 \times 10$ $\mathrm{mm}^{3}$ detector at (a) $20^{\circ} \mathrm{C}$ and (b) $-20^{\circ} \mathrm{C}$ are given in Fig. 1 . The $10.5 \mathrm{keV}$ FWHM energy resolution at $662 \mathrm{keV}$, or $1.59 \%$, obtained at $20^{\circ} \mathrm{C}$ is comparable to the best CPG detectors produced today. Upon cooling to $-20^{\circ} \mathrm{C}$, the energy resolution at $662 \mathrm{keV}$ for this detector improved by more than $40 \%$ to $6.2 \mathrm{keV}(0.94 \%)$. A similar improvement in energy resolution was also observed in a larger $15 \times 15 \times 10 \mathrm{~mm}^{3}$ detector whose spectra are given in Fig. 2.

As a further illustration of the improvement in energy resolution achieved through detector cooling and to demonstrate the reproducibility of the results, we plot in Fig. 3 the $662 \mathrm{keV}$ gamma-ray energy resolution as a function of detector temperature for all of the detectors that we used in this study. This figure summarizes the performance results from seven CdZnTe crystals. Six crystals were processed conventionally and tested. Two of these were then refabricated using our improved process and then retested. Finally, one additional crystal was fabricated using only the improved process and tested. Overall it is clear that decreasing the detector temperature from $20^{\circ} \mathrm{C}$ to $-20^{\circ} \mathrm{C}$ consistently improved the resolution. This improvement ranged from just over $20 \%$ to more than $40 \%$. Furthermore, the reduction occurred regardless of the process used to produce the detector. However, as is evident from Fig. 3, the fabrication method does determine ultimately how well the detector will perform. For the two fabrication processes presented here, the improved process led to resolutions that are roughly $30 \%$ better than those obtained with the conventionally processed detectors.

To further advance the spectroscopic performance of the CdZnTe CPG detectors, it is important to understand the physical basis for the improved energy resolution with reduced detector temperature. To do this, it is instructive to look at the primary detector related contributions to the gamma-ray energy resolution:

$$
\Delta \mathrm{E}_{\gamma}^{2}=\Delta \mathrm{E}_{\mathrm{n}}^{2}+\Delta \mathrm{E}_{\mathrm{d}}^{2}+\Delta \mathrm{E}_{\mathrm{s}}^{2}
$$

Here $\Delta \mathrm{E}_{\mathrm{n}}$ is the broadening from electronic noise; $\Delta \mathrm{E}_{\mathrm{d}}$ is the broadening introduced by non-uniformities in the detector response caused by the underlying material, charge induction, and charge collection non-uniformities; and $\Delta \mathrm{E}_{\mathrm{s}}$ represents the contributions from charge generation and charge trapping statistics. For the large-volume CdZnTe CPG detectors produced today, $\Delta \mathrm{E}_{\mathrm{n}}$ and $\Delta \mathrm{E}_{\mathrm{d}}$ are the dominant contributions, and, in many situations, there can be a trade off between $\Delta \mathrm{E}_{\mathrm{n}}$ and $\Delta \mathrm{E}_{\mathrm{d}}$. For example, by decreasing the pitch between grid strips, the charge collection non-uniformity is reduced since the variation in electron trajectories due to the steering of the electrons to the collecting grid electrode is lessened [8]. The decreased pitch, however, has the opposite effect on the electronic noise because of the increase in the intergrid capacitance. Likewise, increasing the detector biases can reduce the non-uniformity broadening, as discussed in detail below, but this is typically at the price of greater electronic noise.

The widths of the pulser peaks shown in the spectra of Figs. 1 and 2 provide a measure of the electronic noise contribution to the gamma-ray energy resolutions. From the pulser widths it is evident that the electronic noise is reduced by lowering the detector temperature, and that this noise reduction is part of the reason for the improved energy resolution. In order to better understand the physical origin of the noise drop with temperature, we measured the noise as a function of the amplifier peaking time at various temperatures. An example is shown in Fig. 4 where the noise characteristics measured 
from the Fig. 2 detector are plotted. The detector operating conditions for these measurements were chosen to be the same as those used to obtain the spectra of Fig. 2. The symbols plotted in the figure are the measured data points, and the curves are fits to the measured data. The series, 1/f, and parallel components of the noise were extracted as indicated by the straight lines shown in the figure. From this analysis we see that decreasing the temperature from $20^{\circ} \mathrm{C}$ to $-20^{\circ} \mathrm{C}$ has done little to change the series and 1/f noise components yet has substantially reduced the parallel noise. The detector parallel noise is likely dominated by bulk charge injection at the electrical contacts of the detector [12]. This injection process relies on the thermal activation of charge carriers over the potential barrier of the metal-semiconductor interface. Decreasing the detector temperature therefore reduces the charge injection and consequently the parallel noise as we have observed. This explanation is also supported by the observation that the detector leakage currents drop with temperature.

The reduction in the electronic noise with cooling, however, is not the entire explanation for the improved energy resolution at lower temperatures shown in Fig 1 and 2. In fact, as has been observed by Sturm et al. [6], the energy resolution of the detector can actually degrade as the temperature is reduced despite the noise reduction. This is illustrated in Fig. 5 where we plot the gamma resolution and electronic noise as a function of temperature for various bias conditions. The data of Fig. 5 (a) was obtained using a typical cathode bias of $1500 \mathrm{~V}$ and grid biases of 50, 100, and $150 \mathrm{~V}$. For all bias settings, the noise dropped with temperature as expected. The gamma resolution, however, improved initially in going from $20^{\circ} \mathrm{C}$ to $0^{\circ} \mathrm{C}$ but then degraded during a further reduction in temperature to $-20^{\circ} \mathrm{C}$. This increase in the gamma resolution must be the result of degradation in the intrinsic detector properties. One possible mechanism that could lead to this is a decreased effectiveness of the grid bias to deflect the electrons to the collecting grid electrode, which could be caused by a change in the intergrid surface conduction or trapped charge. We would expect, though, that such effects could be lessened through an increase in the grid bias. Since the performance at $-20^{\circ} \mathrm{C}$ was not significantly affected by a grid bias increase from $100 \mathrm{~V}$ to $150 \mathrm{~V}$, this mechanism does not seem to be the source of the degradation.

In contrast, the increase in the intrinsic broadening (gamma-ray resolution with the electronic noise subtracted) observed at $-20^{\circ} \mathrm{C}$ for the cathode bias of $1500 \mathrm{~V}$ was not present when the bias was doubled to $3000 \mathrm{~V}$. This is shown in Fig. 5 (b) by the continuous drop in the gamma resolution with decreasing temperature. At the higher bias, the intrinsic broadening actually decreased when the detector temperature was reduced all the way to $-20^{\circ} \mathrm{C}$. The intrinsic resolutions at $20^{\circ} \mathrm{C}$ for the detectors of Figs. 1 and 2 are both calculated to be about $7.8 \mathrm{keV}$. At $-20^{\circ} \mathrm{C}$, this resolution has decreased to $4.7 \mathrm{keV}$ for the Fig. 1 detector and $5.0 \mathrm{keV}$ for that of Fig. 2. Thus the improved energy resolution at the lower temperature resulted from a combination of the higher operating bias and the decreased electronic noise. It is worth noting that at $20^{\circ} \mathrm{C}$ the use of the larger cathode bias was not beneficial since this necessitated a larger grid bias in order to ensure complete collection to the collecting grid of the gamma-generated charge. The larger noise associated with the increased grid bias produced a poorer overall gamma resolution than that obtained at the lower cathode bias.

The significant improvement in the intrinsic resolution when the cathode bias is increased is an important observation of our study. There are multiple possible mechanisms that could cause this bias dependence. One is the lessening of the impact of the charge trajectory variations as the cathode bias is increased. Ideally it is desired that all gamma-ray interaction events that deposit charge at the same depth within a CPG detector lead to charge collection trajectories of the same path length. When this is the case, the charge induction will be uniform throughout almost the full detector volume. In reality, though, there is variation in the trajectory lengths as a result of the steering of the drifting charge to the collecting grid strips [8]. For example, an event that occurs directly underneath a non-collecting electrode strip will follow a trajectory with a longer path length than one occurring directly underneath a collecting electrode strip and hence will experience more electron trapping. This variation in the amount of charge trapping between events then results in a pulse height variation and a loss of energy resolution. However, by increasing the cathode bias, the electron charge is more rapidly collected, thereby decreasing the overall trapping and reducing the amount of charge loss associated with path length differences. A second mechanism is the reduction in the material-related non-uniform electron trapping. The level of electron trapping in a CdZnTe crystal can spatially vary within the crystal such that the overall trapping experienced by the drifting charge varies from event to event [8]. This situation is much like that of the charge trajectory variations in that the more rapid electron collection caused by a larger cathode bias reduces electron trapping and, consequently, the pulse height variations associated with the event-to-event differences in electron trapping. A third possible mechanism is the lessening of the impact of trapped surface charge on the sides of the detector. Such surface charge can distort the electric field within the detector thereby producing charge trajectory and drift velocity variations. The higher bias voltage tends to straighten the field and reduces the effects of surface charge.

Another interesting finding from our temperature study concerns the CPG spectroscopic performance at more extreme temperatures than those already discussed. From Fig. 3 we see that there was a continuous improvement in energy resolution as the detector temperature was adjusted from near room temperature to $-20^{\circ} \mathrm{C}$. Reducing the temperature much beyond $-20^{\circ} \mathrm{C}$, however, does not appear to be beneficial and actually results in degraded spectroscopic performance. This is illustrated in Fig. 6 where a sequence of ${ }^{137} \mathrm{Cs}$ spectra 
acquired with a CPG detector cooled to $-30^{\circ} \mathrm{C}$ is plotted. The spectra were obtained by placing the source near the detector, waiting a variable length of time prior to starting the data acquisition, and then acquiring pulse height data for a fixed $100 \mathrm{~s}$ interval. Each spectrum in the figure was obtained using a different wait time ranging from $0 \mathrm{~min}$ in (a) to $30 \mathrm{~min}$ in (d). From this sequence of measurements, we see that the spectral performance was initially good, but then degraded over time when exposed to gamma-rays. When the source was removed, the detector performance was observed to recover after a period of minutes, and the measurement could be repeated. This spectroscopic performance degradation was not only observed to be gamma-ray flux dependent but also dependent on detector bias. With a lower cathode bias, the detector exhibited a more rapid loss of performance. The loss of spectral information at temperatures near $-30^{\circ} \mathrm{C}$ was also seen in the other detectors we studied, and similar behavior was noted in the work of Sturm et al. [6].

To identify the cause of the spectral performance degradation, we analyzed the charge signals induced on the detector grid electrodes. A particularly informative set of signal pairs is plotted in Fig. 7. The signals plotted in Fig. 7 (a) are those that would be expected as a result of a gammaray interaction event taking place near the cathode. The collecting and noncollecting grid signals both rose linearly as the gamma-generated electrons drifted towards the grids. When the electrons eventually approached a collecting grid strip, the collecting grid signal increased rapidly at the expense of the noncollecting grid signal. Once the electrons were finally fully collected, the signals became nearly constant except for some minor change due to, for example, collection of detrapped electrons. These signals were obtained from the CPG detector at $-30^{\circ} \mathrm{C}$ immediately after the ${ }^{137}$ Cs source was placed near the detector and indicate that the detector was initially functioning properly. The signals of Fig. 7 (b) in contrast were obtained after the source remained near the detector for $30 \mathrm{~min}$ and are indicative of poor electron collection. These signals show that initially the electron charge drifted through the bulk of the detector as expected. But, at some point, they encountered a weak electric field region, and the electron drift slowed considerably. The slowed collection resulted in more electron trapping and, consequently, a reduced detector signal pulse height. The extent of this slow collection varied widely from event to event and ultimately was the cause of the poor spectroscopic performance.

The existence of a weak electric field region (commonly referred to as polarization) near the grid electrodes must be the result of charge accumulation within this region. A likely source of this charge is the hole charge generated by the gamma rays. At the low temperature, the increased hole detrapping time may cause holes to accumulate in sufficient quantities to affect the field distribution in the detector.

\section{SUMMARY AND CONCLUSIONS}

The results from our CPG detector cooling study carried out with many different CdZnTe crystals and two different fabrication processes demonstrate that improved spectroscopic performance can consistently be achieved through moderate detector cooling. The measurements indicate that improved gamma-ray energy resolution results from a combination of a larger detector bias producing a reduction in the intrinsic broadening and a decreased electronic noise. Under high bias, the energy resolutions of the tested detectors all continued to get better as the detector temperature was decreased until a temperature of about $-30^{\circ} \mathrm{C}$ was reached. At this temperature, a detector bias and radiation flux dependent polarization developed in the detectors that ultimately led to the deterioration of the spectroscopic response.

It is interesting to note from the cooled detector performances obtained in our study how good the CPG performance can be with currently available CdZnTe materials. We extracted an intrinsic peak width at $662 \mathrm{keV}$ of about $5 \mathrm{keV}$ FWHM (0.76 \%) for detectors $1 \mathrm{~cm}^{3}$ and $2.3 \mathrm{~cm}^{3}$ in volume. This is potentially what could be achieved for the total energy resolution through improvements in detector processing and front-end electronics aimed at reducing electronic noise.

\section{REFERENCES}

[1] P. N. Luke, "Single polarity charge sensing in ionization detectors using coplanar electrodes,” Appl. Phys. Lett., vol. 65, pp. 2884-2886 ,1994.

[2] P. N. Luke, "Unipolar charge sensing with coplanar electrodes application to semiconductor detectors," IEEE Trans. Nucl. Sci., vol. 42, no. 4, pp. 207-213, 1995.

[3] Z. He, G. F. Knoll, D. K. Wehe, R. Rojeski, C. H. Mastrangelo, et al., "1-d position-sensitive single carrier semiconductor detectors," Nucl. Instrum. Methods Phys. Res., vol. A 380, pp. 228-231, 1996.

[4] Z. He, G. F. Knoll, D. K. Wehe, and J. Miyamoto, "Position-sensitive single carrier CdZnTe detectors," Nucl. Instrum. Methods Phys. Res., vol. A 388, pp. 180-185, 1997.

[5] V. Gostilo, I. Lisjutin, A. Loupilov, and V. Ivanov, "Performance improvement of large volume CdZnTe detectors," 2004 IEEE Nucl. Sci. Sym. Conf. Rec., vol. 7, pp. 4590-4595, 2004.

[6] B. W. Sturm, Z. He, T. H. Zurbuchen, and P. L. Koehn, IEEE Trans. Nucl. Sci., vol. 52, no. 5, pp. 2068-2075, 2005.

[7] P. N. Luke and E. E. Eissler, "Performance of CdZnTe coplanar-grid gamma-ray detectors," IEEE Trans. Nucl. Sci., vol. 43, no. 3, pp. 14811486, 1996.

[8] P. N. Luke, M. Amman, and J. S. Lee, "Factors affecting energy resolution of coplanar-grid CdZnTe detectors," IEEE Trans. Nucl. Sci., vol. 51, no. 3, pp. 1199-1203, 2004, and references therein.

[9] P. N. Luke, M. Amman, J. S. Lee, and C. Q. Vu, "Pocket-size CdZnTe gamma-ray spectrometer," IEEE Trans. Nucl. Sci., vol. 52, no. 5, pp. 2041-2044, 2005.

[10] eV Products, a division of II-VI, Inc., Saxonburg, PA 16056.

[11] M. Amman, P. N. Luke, and J. S. Lee, "CdZnTe material uniformity and coplanar-grid gamma-ray detector performance," IEEE Trans. Nucl. Sci., vol. 47, no. 3, pp. 760-767, 2000.

[12] P. N. Luke, M. Amman, J. S. Lee, and P. F. Manfredi, "Noise in CdZnTe detectors,” IEEE Trans. Nucl. Sci., vol. 48, no. 3, pp. 282-286, 2001.

[13] T. H. Prettyman, F. P. Ameduri, A. Burger, J. C. Gregory, M. A. Hoffbauer, et al., "Effect of surfaces on the performance of CdZnTe detectors," Proceedings of SPIE, vol. 4507, pp. 23-31, 2001.

[14] M. Amman and P. N. Luke, "Optimization criteria for coplanar-grid detectors,” IEEE Trans. Nucl. Sci., vol. 46, no. 3, pp. 205-212, 1999. 


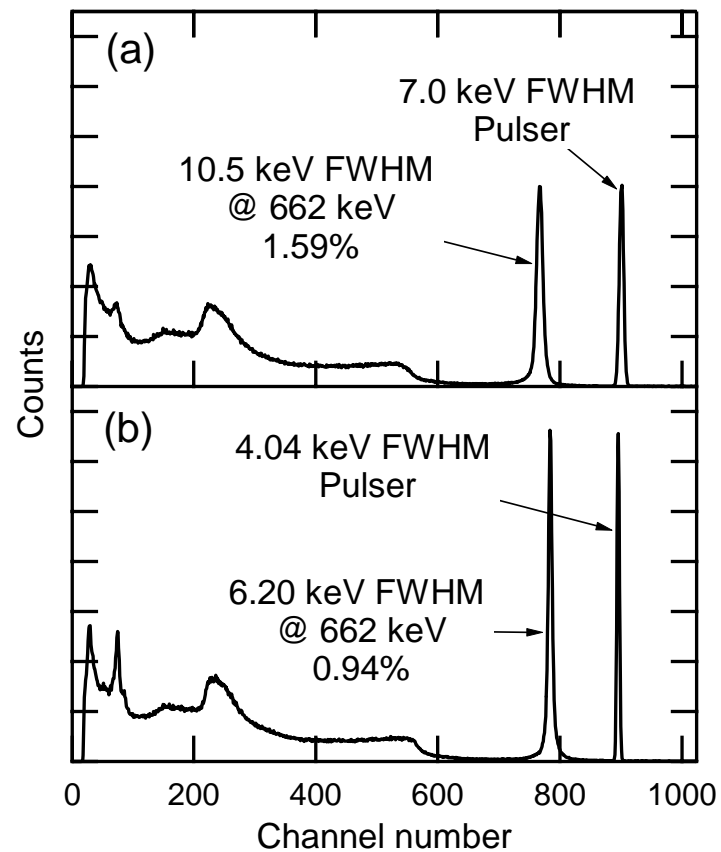

Fig. $1 .{ }^{137}$ Cs gamma-ray pulse-height spectra obtained with a $10 \times 10 \times 10$ $\mathrm{mm}^{3}$ CdZnTe CPG detector when operated near room temperature and with mild cooling. The operating conditions were (a) $\mathrm{T}=20^{\circ} \mathrm{C}, \mathrm{V}_{\mathrm{c}}=1500 \mathrm{~V}$, and $\mathrm{V}_{\mathrm{g}}=50 \mathrm{~V}$ and (b) $\mathrm{T}=-20^{\circ} \mathrm{C}, \mathrm{V}_{\mathrm{c}}=3000 \mathrm{~V}$, and $\mathrm{V}_{\mathrm{g}}=150 \mathrm{~V}$. Both spectra were obtained using a peaking time of $4 \mu \mathrm{s}$.

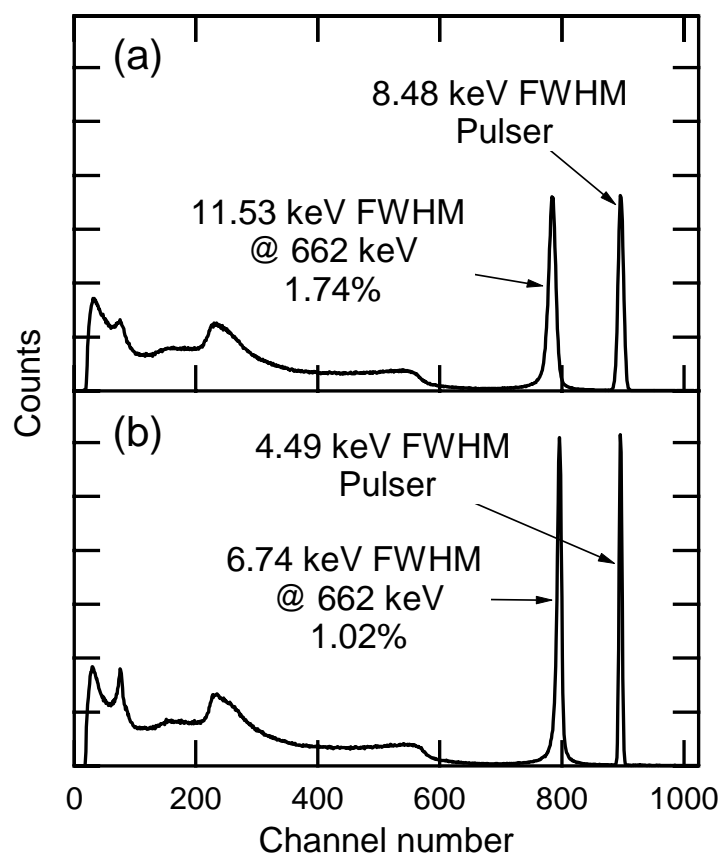

Fig. 2. ${ }^{137} \mathrm{Cs}$ gamma-ray pulse-height spectra obtained with a $15 \times 15 \times 10$ $\mathrm{mm}^{3}$ CdZnTe CPG detector when operated near room temperature and with mild cooling. The operating conditions were (a) $\mathrm{T}=20^{\circ} \mathrm{C}, \mathrm{V}_{\mathrm{c}}=1500 \mathrm{~V}$, and $\mathrm{V}_{\mathrm{g}}=50 \mathrm{~V}$ and (b) $\mathrm{T}=-20^{\circ} \mathrm{C}, \mathrm{V}_{\mathrm{c}}=3000 \mathrm{~V}$, and $\mathrm{V}_{\mathrm{g}}=100 \mathrm{~V}$. Both spectra were obtained using a peaking time of $4 \mu \mathrm{s}$.

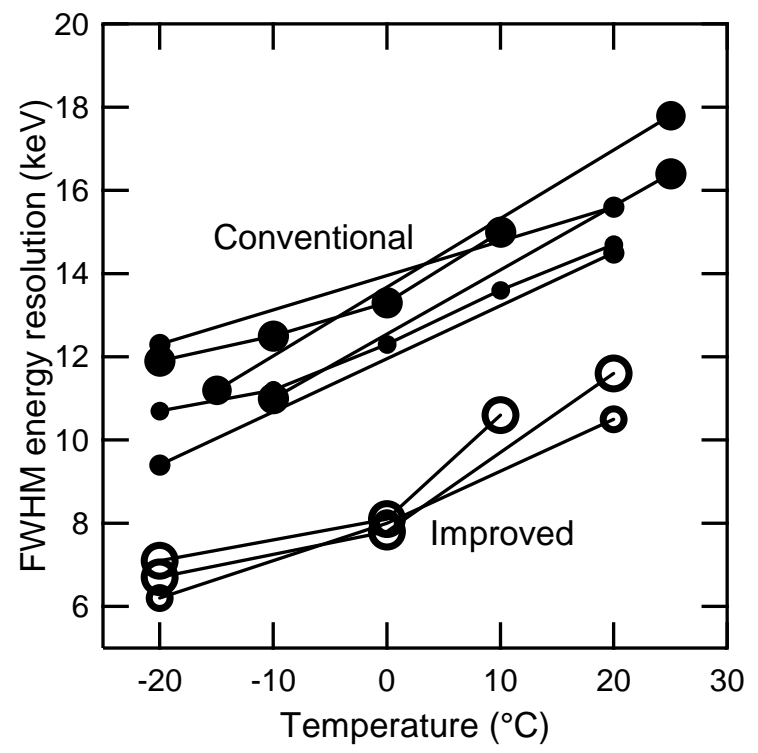

Fig. 3. $662 \mathrm{keV}$ gamma-ray energy resolution measured as a function of temperature with many different CdZnTe CPG detectors. The size of the symbols used is proportional to the detector volume. The smaller symbols represent detectors with an approximate volume of $1 \mathrm{~cm}^{3}$, whereas the larger ones indicate a volume near $2.3 \mathrm{~cm}^{3}$.

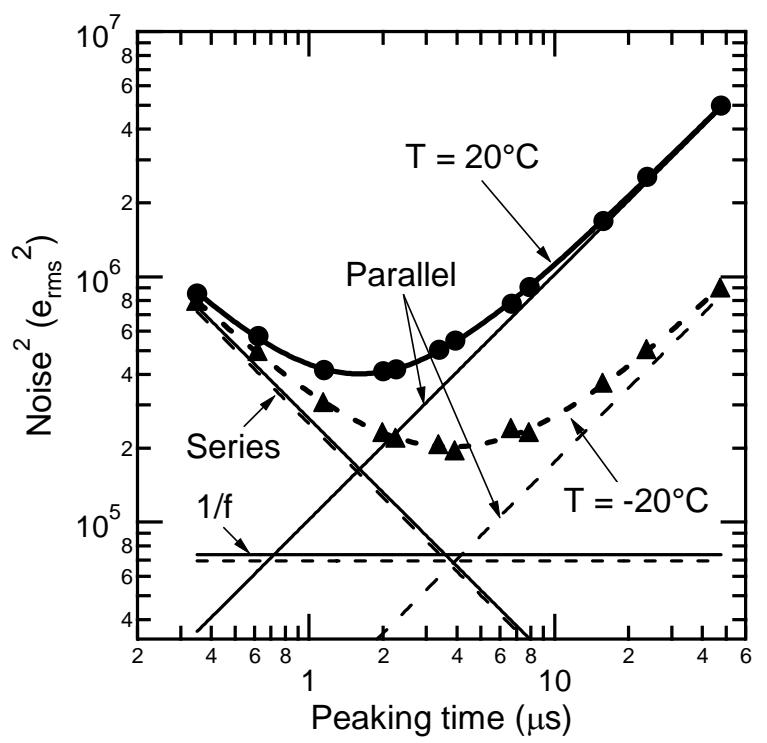

Fig. 4. Measured noise (circular and triangular data points) as a function of amplifier peaking time for the CPG detector used to obtain the spectra of Fig. 2. The curves are obtained by fitting to the data, and the straight lines are the different noise components extracted from the fits. The detector operating conditions were the same as those of Fig. 2. 


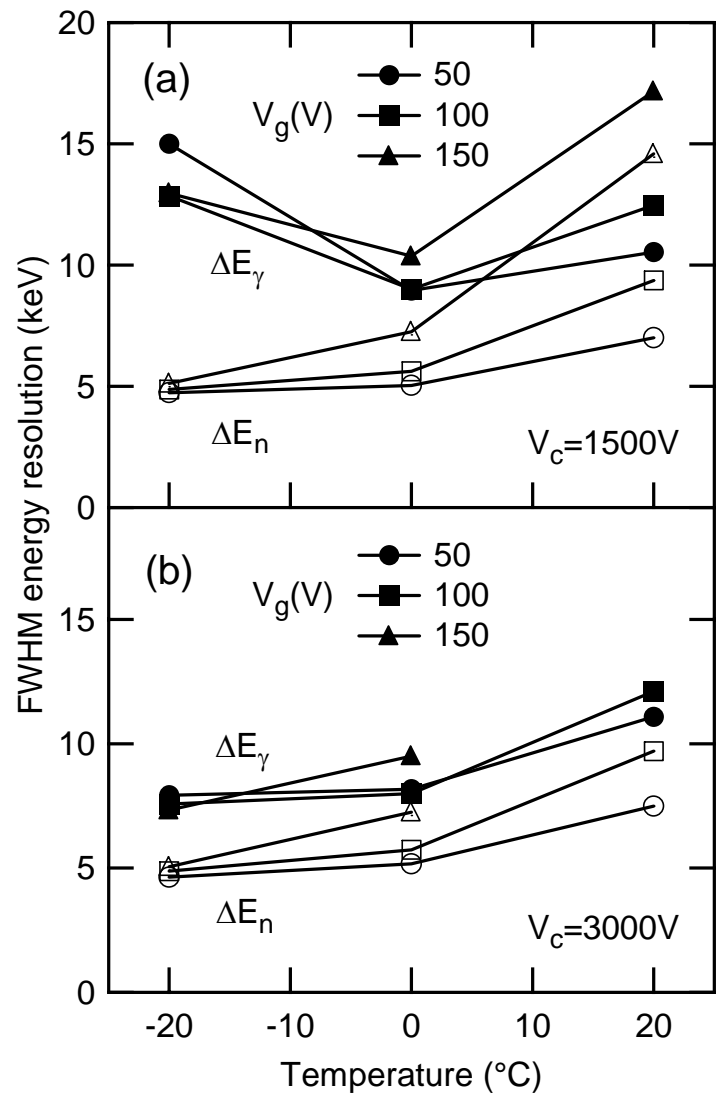

Fig. 5. $662 \mathrm{keV}$ gamma-ray energy resolution and electronic noise extracted from spectra acquired with a $10 \times 10 \times 10 \mathrm{~mm}^{3}$ CdZnTe CPG detector operated at various temperatures and bias settings.

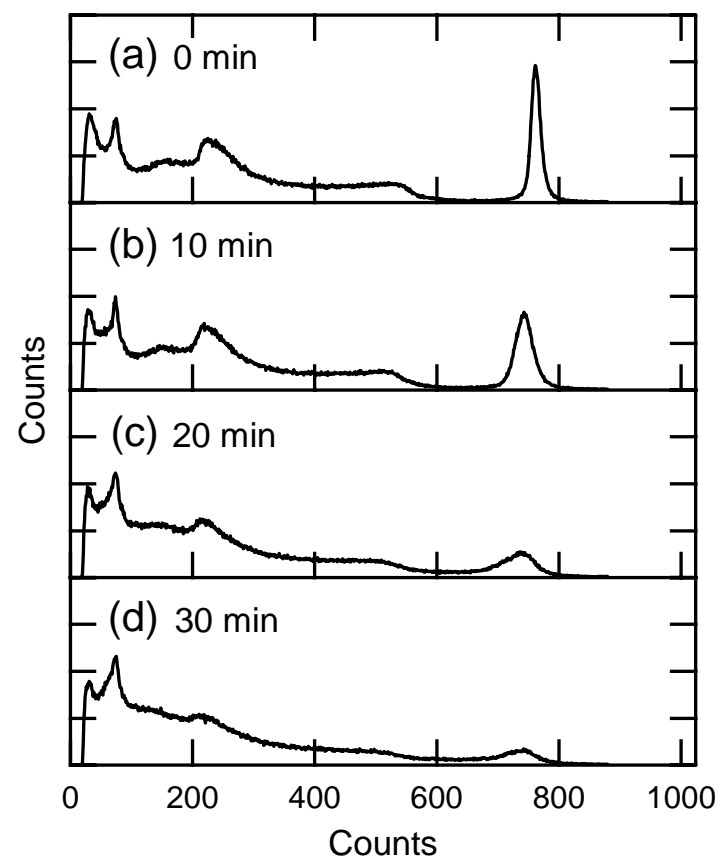

Fig. 6. ${ }^{137} \mathrm{Cs}$ gamma-ray pulse-height spectra obtained with a $15 \times 15 \times 10$ $\mathrm{mm}^{3} \mathrm{CdZnTe}$ CPG detector when operated at $\mathrm{T}=-30^{\circ} \mathrm{C}, \mathrm{V}_{\mathrm{c}}=3000 \mathrm{~V}$, and $\mathrm{V}_{\mathrm{g}}$ $=200 \mathrm{~V}$. For each spectrum, the ${ }^{137} \mathrm{Cs}$ source illuminated the detector for a different length of time prior to the spectrum acquisition. These preacquisition wait times were (a) $0 \mathrm{~min}$, (b) $10 \mathrm{~min}$, (c) $20 \mathrm{~min}$, and (d) $30 \mathrm{~min}$. The typical event count rate was about 4700 counts/s and is comparable to that used to obtain the data of Figs. 1 - 3.

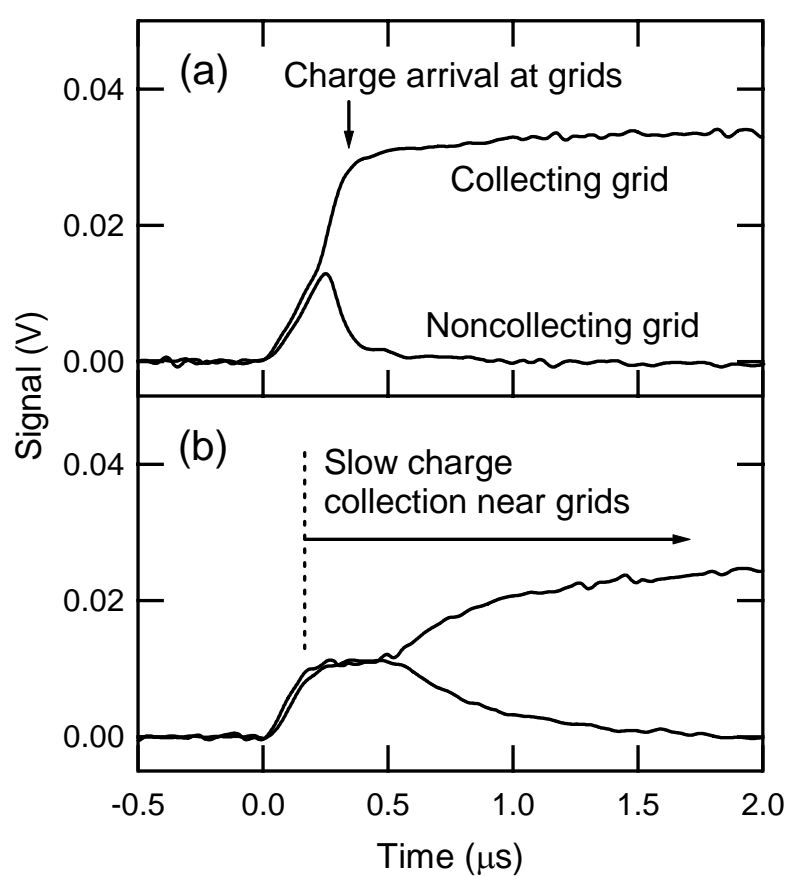

Fig. 7. Induced charge signals measured on the grid electrodes of the CPG detector used to obtain the spectra of Fig. 6. The detector operating conditions were the same as those of Fig. 6. The signals result from the collection of the charge deposited by a $662 \mathrm{keV}$ gamma ray interaction event near the cathode. Event (a) was acquired shortly after the ${ }^{137} \mathrm{Cs}$ source illuminated the detector, and (b) was acquired after a 30 min delay. 